Technologies in Health (CADTH) was selected a priori as data source for this review of systematic reviews. The review was limited to high quality SRs of interventions targeting clinicians.

Results A total of 12 SRs met study inclusion criteria. These SRs suggest that implementation strategies, such as audit and feedback, academic detailing, and educational meetings, are generally effective in improving providers' behaviours, with small to moderate effect sizes.

Discussion This review of SRs provides support for the overall efficacy of guideline implementation strategies, while highlighting the need for further comparative and cost effectiveness research to address gaps in the knowledge identified (e.g., limited information on head-to-head comparisons between strategies, clinical context, and cost of interventions).

Implications for Guideline Developers/Users Guideline developers should include recommendations for guideline implementation in their future guidelines. Making specific recommendations on choosing one implementation strategy over the others should be avoided until further head-to-head comparisons are available.

\section{P217 THE EFFECT OF PRINT OR ONLINE EDUCATIONAL MATERIALS FOR PRIMARY CARE PHYSICIANS: A SYSTEMATIC REVIEW}

${ }^{1,2} \mathrm{~A}$ Grudniewicz, ${ }^{3} \mathrm{R}$ Rodseth, ${ }^{1} \mathrm{R}$ Kealy, ${ }^{1} \mathrm{D}$ Rudoler. 'University of Toronto, Toronto,
Canada; ${ }^{2}$ Li Ka Shing Knowledge Institute, St. Michael's Hospital, Toronto, Canada; ${ }^{3}$ University of KwaZulu Natal, Durban, South Africa

\section{0:1136/bmjqs-2013-002293.211}

Background Print and online materials such as guideline summaries are commonly used to distribute evidence to primary care physicians; they are easy to implement and scale across many primary clinics.

Objectives We sought to determine: 1) if providing primary care physicians with print and online educational materials has an effect on physician behaviour or on patient outcomes, 2) how these materials were developed, and 3) whether design attributes impact outcomes.

Methods We systematically identified studies that reported a print or online educational intervention for primary care physicians. Studies were identified by searching four electronic databases, scanning reference lists, and contacting experts. A subanalysis was conducted to collect data on how these materials were developed and on their use of design principles.

Results Thirty studies met eligibility criteria after full-text screening. Studies targeted physician advice-giving behaviour, diagnostic procedures, prescribing behaviour, change in knowledge, and clinical patient outcomes. Results suggest that print and online materials targeted at primary care physicians have little to no effect on outcomes.

Discussion Print and online educational materials provided to primary care physicians have little effect on physician or patient outcomes. This is concerning as they are a common method of disseminating evidence. Most studies do not describe how interventional materials were developed or whether design principles were applied.

Implications for Guideline Developers/Users Design principles should be considered when developing evidence-based materials and the development processes should be described in order to determine if better designs influence uptake and use of evidence.

\section{P219 ENGAGING CONSUMERS IN THE GUIDELINE DEVELOPMENT PROCESS - THE US PERSPECTIVE}

P Robertson, H Hussey, S Jones. American Academy of Otolaryngology-Head and Neck Surgery Foundation, Alexandria, USA

10:1136/bmjqs-2013-002293.212

Background In the United States, there have been increasing calls for guideline developers to engage consumers throughout the guideline development process. The Guidelines International Network (G-I-N) and the Institute of Medicine (IOM) have both released guideline development best practices encouraging consumer involvement; ranging from consumer input during the formulation of clinical questions, to serving as a guideline panel member and participating in the review process.

Context Our organisation has been developing evidence based clinical practice guidelines for nearly a decade and has incorporated consumers in the development process for over five years. By including consumers, our guidelines now feature more patient-centred recommendations; establishing a more balanced discussion of patient preferences and improving how we assess benefits and harms.

Description of Best Practice To more readily identify consumers for guideline development, our organisation has built a collaborative relationship with a consumer advocacy alliance. Through this relationship, we have been able to support two consumer advocates as full members on each guideline panel. To assist their participation, we provide education about our guideline development process, and outline the expectations of their involvement throughout the process.

Lessons for Guideline Developers Our experience with consumer engagement can serve as an example for other US guideline developers. Consumers can bring invaluable insight and perspective throughout guideline development and have substantially improved our guidelines. We believe consumer participation will become increasingly important in the coming years, particularly as guideline developers move towards a standardised approach to development.

\section{P222 EVOLUTION OF EVIDENCE GRADING SYSTEMS IN THE AMERICAN ACADEMY OF OPHTHALMOLOGY'S PREFERRED PRACTICE PATTERNS}

N Emptage, F Lum. American Academy of Ophthalmology, San Francisco, USA

\section{0:1136/bmjqs-2013-002293.213}

Background Clinical practice guidelines are an important component of efforts to improve quality of care and rationalise the introduction of new medical technologies. As evidence-based medicine and comparative effectiveness research become more prominent, the rigour and transparency of guideline development is becoming increasingly important.

Context Since 1988, the American Academy of Ophthalmology has published ophthalmic practice guidelines known as Preferred Practice Patterns (PPPs). Over time, the Academy has introduced increasingly rigorous processes for grading the evidence underpinning the PPPs.

Description of Best Practice Prior to 2000, the PPPs were effectively consensus-based, with no formal processes for identifying or synthesising evidence, and no system for grading evidence quality. In 2000, a three-level system was introduced to denote the quality of the evidence supporting PPP recommendations, 